\title{
Flavor Filtered Fermions
}

\section{James C. Osborn*}

Leadership Computing Facility

Argonne National Laboratory

9700 S. Cass Ave.

Argonne, IL 60439, USA

E-mail: osbornealcf.anl.gov

\section{Xiao-Yong Jin}

Leadership Computing Facility

Argonne National Laboratory

9700 S. Cass Ave.

Argonne, IL 60439, USA

E-mail: xjin@anl.gov

\begin{abstract}
We present a new discretization of the lattice Dirac operator that preserves chiral symmetry while suppressing the effects of doubler states. The new operator is constructed from the naive Dirac operator by filtering out doubler states while preserving one physical quark state. In the simplest version the filtering is only approximate and the filter would require fine tuning to produce a single flavor in the continuum limit. We will present results from quenched simulations for the doubler suppression, locality and topology of the operator. We also discuss prospects for improving the filter to reduce or possibly eliminate the need for fine tuning.
\end{abstract}

The 33rd International Symposium on Lattice Field Theory

14 - 18 July 2015

Kobe International Conference Center, Kobe, Japan*

${ }^{*}$ Speaker. 
Discretizing the Dirac operator on a space-time lattice has historically led to a series of compromises. The naive discretization leads to extra doubler fermion states that become extra physical flavors of fermions in the continuum limit. To overcome this Wilson [1] added an extra term to the naive operator that made the doublers heavy and thus decouple from the theory, but broke chiral symmetry in the process. Another option emerged with the rediscovery of an idea put forth by Ginsparg and Wilson (GW) [2] which allowed for a single flavor of fermion to only mildly break chiral symmetry, while remaining local. The GW relation is satisfied by the domain wall (approximately) [3] and overlap [4] operators, which were later discovered to be related. These GW Dirac operators offer theoretically nice properties with the major downside being a significantly increased computational cost compared with the simpler, but less theoretically pleasant alternatives.

Here we present a new discretization of the Dirac operator that also preserves chiral symmetry in accordance with the Ginsparg-Wilson relation but is computationally much less expensive than the other formulations that do. However the elimination of the doubler states is only approximate in the simplest implementation. While the doublers are still present, they are suppressed compared to the one wanted flavor. We have explored simple tuning of the filter for valence measurements and will also discuss further directions for improvement. We also mention the possibility of applying the same approach to create a 2 flavor operator from staggered fermions.

\section{Flavor Filtered Fermions}

The naive discretization of the massless Dirac operator is

$$
D_{N}=\frac{1}{2} \sum_{\mu} \gamma_{\mu}\left[T_{\mu}-T_{\mu}^{\dagger}\right]
$$

using the parallel transporter for direction $\mu$

$$
T_{\mu}(x, y)=U_{\mu}(x) \delta_{x+a \hat{\mu}, y}
$$

with $a$ the lattice spacing. In the free case $\left(U_{\mu}=1\right)$ the naive Dirac operator in momentum space is

$$
D_{N}(p, q)=\frac{1}{V} \sum_{x, y} \mathrm{e}^{-i p \cdot x} D_{N}(x, y) \mathrm{e}^{i q \cdot y}=\delta(p, q) i \sum_{\mu} \gamma_{\mu} \sin \left(a p_{\mu}\right) .
$$

This operator has 16 zeros at momenta points where $a p_{\mu} \in\{0, \pi\}$. We will refer to the mode at $p=\{0,0,0,0\}$ as the wanted physical one and the 15 other modes as unwanted doublers. In the interacting case, there will still be 16 poles in the propagator, which will become 16 quark species in the continuum limit.

Here we will attempt to eliminate these states from the propagator by multiplying it by some operator $F$ to get

$$
F\left(D_{N}\right)^{-1} F^{\dagger}
$$

with the above form chosen to preserve $\gamma_{5}$-Hermiticity and chiral symmetry. The operator $F$ should filter out the doubler flavors while preserving the physical flavor. One possible choice for the "flavor filter" in momentum space is

$$
F=\prod_{\mu}\left[1+\cos \left(a p_{\mu}\right)\right] / 2 .
$$


One can see that this operator is 1 for $p=\{0,0,0,0\}$ and zero if any of the $a p_{\mu}=\pi$.

If we were to define our new lattice Dirac operator as the inverse of the propagator in (1.4), we would get divergences when the filter goes to zero at the doubler states. We can alleviate this problem by appealing to the Ginsparg-Wilson relation

$$
\frac{1}{D} \gamma_{5}+\gamma_{5} \frac{1}{D}=\gamma_{5} \rho+\rho \gamma_{5}
$$

where $\rho$ is some local operator which we will take to be the constant $1 / 2$. We then define our new propagator to be

$$
D_{F}^{-1}=\rho+F\left(D_{N}\right)^{-1} F^{\dagger} .
$$

After transforming the filter $F$ back to real space and including the gauge field, we get

$$
F=\operatorname{Sym}_{\mu} \prod_{\mu}\left[2+T_{\mu}+T_{\mu}^{\dagger}\right] / 4
$$

where $\operatorname{Sym}_{\mu}$ means to symmetrize the product over all permutations of $\mu$ in order to preserve rotational invariance. Since the links will effectively be renormalized, we instead consider a more general form of the filter given by replacing the individual terms in the product with

$$
F_{\mu}=(1-\alpha)+\frac{\alpha}{2}\left(T_{\mu}+T_{\mu}^{\dagger}\right)
$$

This allows us to explore the transformation from the naive operator $(\alpha=0)$ to the tree level flavor filtered one $(\alpha=0.5)$ and to provide a simple approach to tuning the filter $(\alpha>0.5)$.

If we expand the filter into a set operators with one for each number of links in the product, we would get 5 operators corresponding to a 0 link (constant) operator through a 4 link operator. These 5 terms would correspond to the leading order (dimension 3) operators that appear in the corresponding Symanzik effective action. The general case for tuning at leading order would then require 5 parameters. Since the overall normalization can be accounted for by scaling observables, only ratios of the coefficients need to be fine-tuned, which leaves 4 parameters. The feasibility of tuning these parameters is currently under study.

The massless Dirac operator corresponding to the propagator (1.7) is

$$
D_{F}=\frac{1}{\rho}-\frac{1}{\rho} F\left[\rho D_{N}+F^{\dagger} F\right]^{-1} F^{\dagger} .
$$

The simplest way to include a mass is to replace $D_{N}$ with $D_{N}+m$. This has the advantage that one can spin-diagonalize the denominator in the propagator to get 4 staggered Dirac matrices so the effort for the solver is just that of 4 staggered solves. One could use other operators for the mass term by multiplying $m$ by some operator $G$ that, for example, would make the doublers heavier than the physical mode. However this may increase the expense of the operator making the solver more expensive. One would need to weigh the possibly improved condition number of the operator with the increased cost to determine if it is worthwhile. For now we stick to the simplest mass term.

We can get an alternate picture of how the doubler states are being filtered out by looking at the action. The fermion determinant can be rewritten as the ratio of determinants

$$
\left|D_{F}\right|=\frac{\left|D_{N}+m\right|}{\left|\rho\left(D_{N}+m\right)+F^{\dagger} F\right|} .
$$



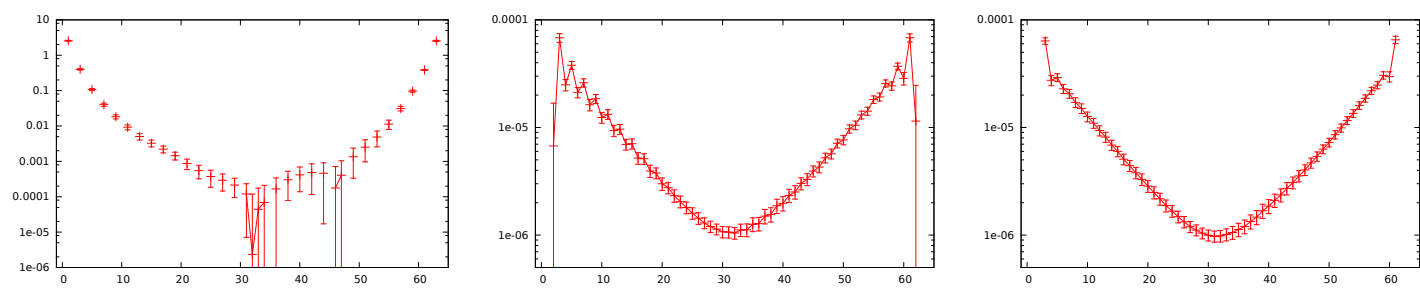

Figure 1: Local pion $\gamma_{4} \gamma_{5}$ correlator on quenched $32^{3} \times 64$ lattices with varying filter parameter $\alpha$ of 0 (left), 0.5 (center) and 0.54 (right). For $\alpha=0$ there is a large contribution from the oscillating state which makes the correlator negative on even time slices and therefore not shown on the plot. At $\alpha=0.54$ the oscillating state is too small to measure.

The numerator contains the usual naive Dirac matrix, which will give 16 flavors of quarks in the continuum. The denominator corresponds to a set of 16 bosonic (ghost) quarks that, in the absence of the extra $F^{\dagger} F$ term, would quench out the regular fermionic quarks leaving a fully quenched theory. The extra filter term, however, makes one of the 16 bosonic quarks heavy while keeping the other 15 light, so that only the light 15 bosonic quarks quench out 15 of the fermionic quarks leaving a single light fermionic quark and the heavy bosonic quark, which decouples from the theory. This partial quenching of 15 of the 16 flavors, however, is only approximate without a properly tuned filter.

\section{Simulation results}

We performed tests on quenched gauge field configurations generated with a standard plaquette action on $32^{3} \times 64$ lattices with $\beta=6.4$. The meson spectrum measurements use a lattice quark mass of $a m=0.0069$ which gives a pion mass in lattice units of about $a m_{\pi} \approx 0.15$. The flavor filtered fermion operator was implemented in the FUEL code [5].

In Figure 1 we show results for the pion spectrum using the local pion operator $\bar{\psi}(x) \gamma_{4} \gamma_{5} \psi(x)$ for a point source. This operator has an oscillating state propagating in time that is very large in the case of naive fermions $(\alpha=0)$, and is not visible on the plot since it makes the correlator on the even time slices go negative. When the filter is at $\alpha=0.5$ the oscillating state has been noticeably suppressed, and for $\alpha=0.54$ it is no longer visible in the correlator.

In Figure 2 we show non-local meson correlators which are obtained by including combinations of shifts and phase factors in the source and sink operators. The sources were constructed from 8 wall sources confined to even or odd sites in each spatial dimension. For naive fermions, the propagator reduces to 4 copies (flavors) of staggered fermions. We can then identify states using the staggered (spin $\otimes$ taste) language. We prefix this notation with an extra factor that indicates the flavor state due to the 4 staggered copies and will use this language to refer to the filtered operator states too. The nonlocal meson states we show here correspond to the operator $\gamma_{F}\left(\gamma_{45} \otimes \xi_{45}\right)$ where $\gamma_{F}$ is given in the plots. The notation $F_{45}$ from the plot corresponds to a $\gamma_{45}$ for flavor, and gives the local pion operator used in Figure 1. This meson is comprised of just the physical quarks. The other flavor operators correspond to mesons that have at least one doubler quark and are therefore suppressed by the filter. We can see the level of suppression of these doubler mesons vary as $\alpha$ is changed, with the best suppression happening around $\alpha=0.5352$. 

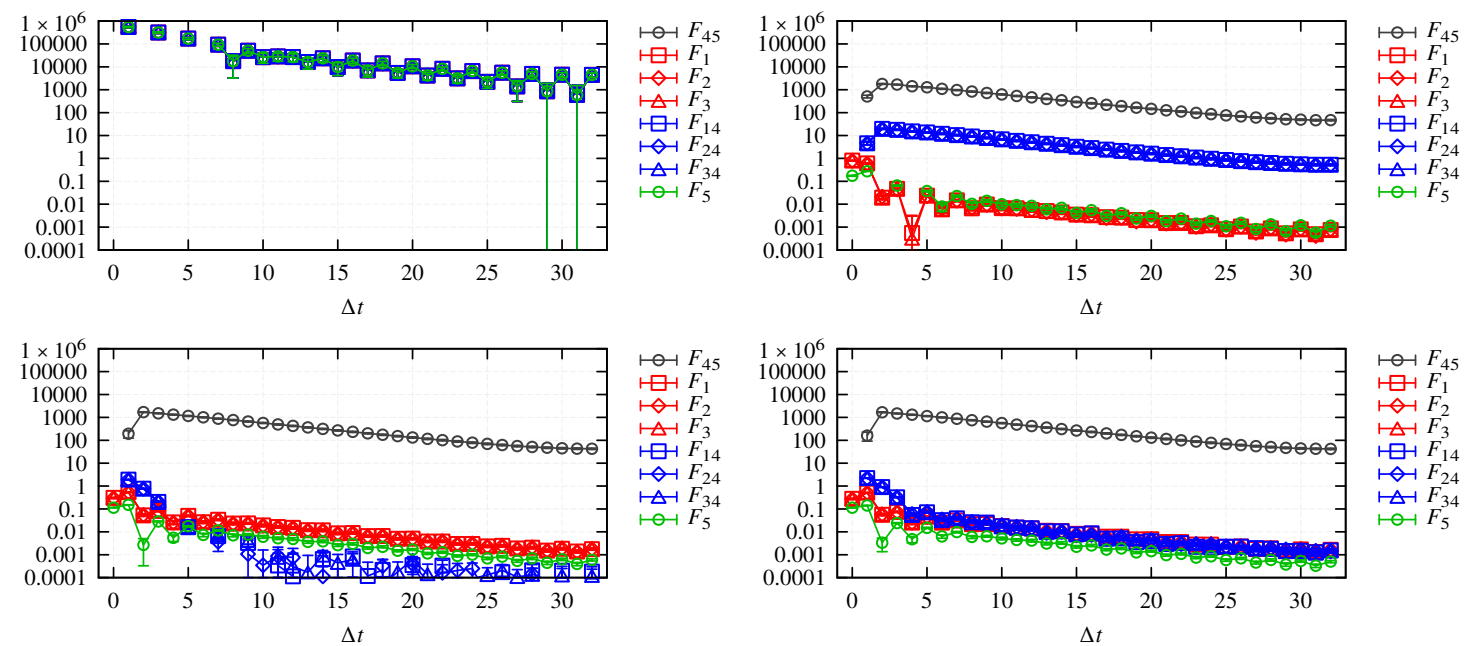

Figure 2: Non-local meson correlator with staggered flavor(spin $\otimes$ taste) identification $\gamma_{F}\left(\gamma_{45} \otimes \xi_{45}\right)$ on quenched $32^{3} \times 64$ lattices with varying filter parameter $\alpha$ of 0 (top left), 0.5 (top right), 0.5352 (bottom left) and 0.54 (bottom right).

We can quantify the level of suppression of the doubler mesons by comparing the amplitude of the exponential decay in the correlators to that of the physical meson state. For the untuned filter $(\alpha=0.5)$ from Figure 2 we see a suppression in the amplitude of about $10^{-2}$ for the $F_{i 4}$ states. The suppression of these states improves to about $10^{-4}$ at $\alpha=0.54$, however there are other states not shown that have a suppression of only about $3 \times 10^{-4}$. With further tuning we can get a suppression of about $10^{-4}$ at $\alpha=0.5352$ for all the possible states that would be identified as pions in the staggered (naive) basis.

The previous results are all for quenched lattices with a plain plaquette action. If we smooth the lattice with 2 steps of stout smearing [6] we now find a suppression of $3 \times 10^{-4}$ at $\alpha=0.5$ and $10^{-6}$ at $\alpha=0.50615$. From this we see that using a single parameter to tune the suppression of doublers works very well for valence measurements. However, for valence measurements, the particular choice of the source will affect the precise value of $\alpha$ for optimal tuning. Tuning the suppression of doublers in a way that is appropriate for the action in dynamical simulations is presently under study.

In Figure 3 (left) we plot the decay of the filtered Dirac operator at $\alpha=0.5$ in the time direction for the unsmeared (nstout=0) and 2 step stout smeared (nstout=2) lattices. This is obtained from applying the Dirac operator to a point source and then taking the magnitude of the result at a point offset by the given number of sites in the time direction. We can see a steep initial drop at short distances followed by a long tail due to the incomplete suppression of the doubler modes. The stout smearing helps suppress the tail. The suppression of the long range tail in the Dirac operator should correlate with the suppression of the doubler modes from the propagator. For a given lattice size, the filter would need to be tuned well enough to suppress the effects of the tail to a negligible level. The exact level of suppression needed for common observables has not been studied yet.

In Figure 3 (right) we show the smallest magnitude eigenvalues of the filtered Dirac operator with $m=0.01$ on a $8^{4}$ quenched lattice at $\beta=5.8$. The black x's are for $\alpha=0$ and are fourfold degenerate as expected for the naive Dirac operator. The blue circles are for $\alpha=0.54$ and 

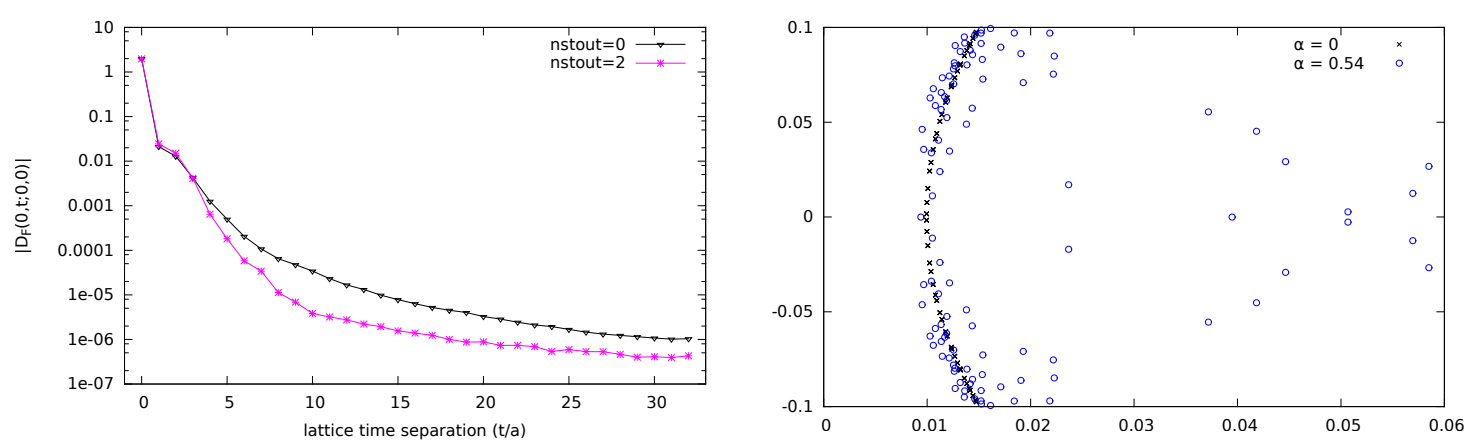

Figure 3: Left: Magnitude of filtered $(\alpha=0.5)$ Dirac operator at a site separated from a point source in the time direction on quenched $32^{3} \times 64$ lattices with a relatively small quark mass $(m=0.001)$. The triangles are without smearing and the bursts are with two steps of stout smearing. Right: Smallest eigenvalues of $D_{F}$ at $m=0.01$ for $\alpha=0$ (naive fermion limit) and $\alpha=0.54$ on a $8^{4}$ quenched lattice. The $\alpha=0.54$ eigenvalues are scaled to overlap with the $\alpha=0$ points. There are 2 real eigenvalues with values of about 0.01 and 0.04 . The larger real eigenvalue is surrounded by 14 other eigenvalues that have moved away from the main curve of eigenvalues towards larger real values.

are multiplied by an overall scale factor to have the bulk of the eigenvalues fall near the naive eigenvalue curve. The eigenvalues spread out in the complex plane due to the nonzero mass. This allows some of the eigenvalues to move towards the set of doubler eigenvalues that should accumulate with a real part of around 2 (which are not shown here). This configuration can be identified as having topology $|Q|=1$ as seen by the real eigenvalue emerging near 0.01 in the filtered operator. This behavior of the eigenvalues might allow $D_{F}$ to exhibit a single small real eigenvalue in the continuum (with careful tuning), in agreement with the index theorem, by shifting the other 15 doubler eigenvalues to have large real parts. Further study is needed to verify if this is the case.

We have also explored the possibility of finding improved forms for the filter that can reduce the effects of doublers with minimal need for tuning. We have considered various filters with more hops in each direction. One example is to use the Wilson term to form a filter

$$
W=4-\frac{1}{2} \sum_{\mu}\left(T_{\mu}+T_{\mu}^{\dagger}\right)
$$

and construct a polynomial from this that is near 1 for small eigenvalues of $W$ and near 0 for larger eigenvalues. Having an abrupt jump would make the filter more non-local so we tried forms with a smooth drop from 1 to 0 . A 64th order polynomial was able to suppress the the doubler meson states by at least a factor of $10^{-6}$ so that their correlators were too noisy to measure accurately. However this form of filter noticeably effects the short range behavior of the correlators and is likely not suitable for general use. We are instead exploring other versions of improved filters.

One might ask if there is a form of the filter at any cost that could give optimal suppression of the doublers without any tuning necessary. In fact we can easily construct one if we consider a modified version of the propagator given by

$$
\rho+F \frac{1 / 2}{D_{N}+m}+\frac{1 / 2}{D_{N}+m} F^{\dagger}
$$


For $m=0$ we can choose a filter containing the overlap Dirac operator

$$
F=\left[\frac{1}{D_{\text {overlap }}}-\rho\right] D_{N}
$$

and the propagator will reduce to exactly the overlap propagator. Using the overlap operator in the filter saves no effort over using an overlap propagator directly, but there may be a cheaper approximation of this filter that provides an effective approximation of the overlap propagator. This is an approach we are currently pursuing.

\section{Flavor (Taste) Filtered Staggered Fermions}

We can apply the same construction presented here to staggered fermions instead of naive. One simple way is to use a filter of the form $F=(1-\alpha)+\alpha\left(1 \otimes \xi_{5}\right)$ in the staggered spin-taste language similar to the operator used in staggered-Wilson fermions [7]. This will filter the 4 staggered tastes down to 2. Unfortunately finding a filter that can produce 1 flavor is problematic since the obvious candidates break rotational symmetry [8].

\section{Summary}

We have presented a new form of lattice Dirac operator that satisfies the Ginsparg-Wilson relation. The corresponding propagator is much cheaper to calculate than for overlap or domain wall fermions. However it does not completely remove all the doubler states without fine tuning or an expensive calculation in the filter. The prospects for improving the filter and making the operator match the overlap operator better with minimal cost are currently being studied. The operator also appears to be sensitive to the global topology and might be able to reproduce the index theorem. The same construction can also be applied to the staggered Dirac operator to create an even cheaper operator that could approach two quark flavors in the continuum limit.

Acknowledgments - This work was supported in part by and used computational resources of the Argonne Leadership Computing Facility, which is a DOE Office of Science User Facility supported under Contract DE-AC02-06CH11357. X.-Y. Jin was also supported in part by the DOE SciDAC program.

\section{References}

[1] K. G. Wilson, New Phenomena In Subnuclear Physics, ed. A. Zichichi (Plenum Press, New York), Part A (1977) 69.

[2] P. H. Ginsparg and K. G. Wilson, Phys. Rev. D 25 (1982) 2649.

[3] D. B. Kaplan, Phys. Lett. B 288 (1992) 342.

[4] H. Neuberger, Phys. Lett. B 417 (1998) 141.

[5] J. C. Osborn, PoS (LATTICE2014) 028; http://jcosborn.github.io/qhmc/.

[6] C. Morningstar and M. Peardon, Phys. Rev. D 69 (2004) 054501.

[7] D. H. Adams, Phys. Lett. B 699 (2011) 394.

[8] C. Hoelbling, Phys. Lett. B 696 (2011) 422. 